\title{
Squeezed vacuum as an eigenstate of two-photon annihilation operator
}

\section{L MEHTA, ANIL K ROY*' and G M SAXENA**}

Department of Physics, Indian Institute of Technology, New Delhi 110016, India

* Centre for Theoretical Studies, Indian Institute of Science, Bangalore 560012, India

** Time and Frequency Section, National Physical Laboratory, New Delhi 110012, India

MS received 22 November 1993

Abstract. We introduce the inverse annihilation and creation operators $\hat{a}^{-1}$ and $\hat{a}^{\dagger-1}$ by their actions on the number states. We show that the squeezed vacuum $\exp \left(\frac{1}{2} \xi a^{\dagger 2}\right]|0\rangle$ and squeezed first number state $\exp \left[\frac{1}{2} \xi a^{\dagger 2}\right]|n=1\rangle$ are respectively the eigenstates of the operators $\left(a^{+-1} a\right)$ and $\left(a a^{\dagger-1}\right)$ with the eigenvalue $\xi$.

Keywords. Coherent states; squeezed states; squeezed vacuum; two-photon annihilation operators.

\section{PACS No. $42 \cdot 50$}

\section{Introduction}

There are several representations of the harmonic oscillator states. The extensively studied states are the number states, the coherent states [1] and the squeezed states [2-6]. The squeezed states are particularly important due to their potential application in noise reduction.

The number states are the eigenstates of the number operator $\hat{a}^{\dagger} \hat{a}$ :

$$
a^{\dagger} a|n\rangle=n|n\rangle, \quad n=0,1,2, \ldots,
$$

whereas the coherent states are the eigenstates of the annihilation operator $a$ :

$$
\hat{a}|\alpha\rangle=\alpha|\alpha\rangle \text {. }
$$

Here $a$ and $a^{\dagger}$ are the boson annihilation and creation operators satisfying the commutation relation $\left[a, a^{\dagger}\right]=1$ and $\alpha$ is an arbitrary complex number.

It is interesting to note that the ground state $|0\rangle$ corresponding to $n=0$ is also a coherent state with $\alpha=0$. This state, also called the vacuum state, is an eigenstate of the annihilation operator $\hat{a}$ with eigenvalue zero. A unitarily transformed vacuum $\hat{U}|0\rangle$ will then be an eigenstate of the unitarily transformed operator $\hat{U} a \hat{U}^{\dagger}$ with zero eigenvalue:

$$
\hat{U} a \hat{U}^{\dagger}(\hat{U}|0\rangle)=0 .
$$

Here we have used the unitarity relation of the operator $\hat{U}$ :

$$
\hat{U}^{\dagger} \hat{U}=\hat{U} \hat{U}^{\dagger}=I \text {. }
$$




\section{L Mehta et al}

If we choose $\hat{U}$ to be the displacement operator

$$
\hat{D}(\alpha)=\exp \left(\alpha \hat{a}^{\dagger}-\alpha^{*} \hat{a}\right)
$$

we get the coherent state eigenvalue equation

$$
(a-\alpha)|\alpha\rangle=0 \text {. }
$$

Similarly, if we choose $\hat{U}$ to be the squeeze operator

we obtain

$$
\hat{S}(\sigma)=\exp \left[\frac{1}{2}\left(\sigma \hat{a}^{\dagger 2}-\sigma^{*} a^{2}\right)\right]
$$

$$
\left(\hat{a} \cosh r-a^{\dagger} e^{i \phi} \sinh r\right)|\sigma, 0\rangle=0,
$$

where we have written $|\sigma, 0\rangle$ for squeezed vacuum, i.e.,

and

$$
|\sigma, 0\rangle=\hat{S}(\sigma)|0\rangle
$$

$$
\sigma=r e^{l \phi} \text {. }
$$

While (1.6) can be expressed as the eigenvalue equation $a|\alpha\rangle=\alpha|\alpha\rangle$, we note that such a separation is not possible for (1.8), where the operator could be made independent of the parameter $\sigma$.

In this paper we aim to obtain a new eigenvalue equation for the squeezed vacuum which does not have the above mentioned limitation. Our motivation is, therefore, to look for an operator independent of $\sigma$ of which the state $|\sigma, 0\rangle$ is an eigenstate. In fact, we consider a slight generalization: In place of the usual squeeze operator (1.7) we let the exponential of a general quadratic $\left[\frac{1}{2}\left(\sigma a^{\dagger 2}-\sigma^{*} a^{2}\right)+i \beta\left(a^{\dagger} a+a a^{\dagger}\right)\right]$ act on to the vacuum. By making use of the normal ordered form of this exponential operator, we may express the state

$$
\exp \left[\frac{1}{2}\left(\sigma a^{\dagger 2}-\sigma^{*} a^{2}\right)+i \beta\left(a^{\dagger} a+a a^{\dagger}\right)\right]|0\rangle
$$

in the form [7]

with

$$
\left(1-|\xi|^{2}\right)^{1 / 4} \exp \left(\frac{1}{2} \xi a^{\dagger 2}\right)|0\rangle
$$

$$
\xi=\frac{\sigma}{(\lambda \operatorname{coth} \lambda-2 i \beta)}
$$

where $\lambda^{2}=\left(|\sigma|^{2}-4 \beta^{2}\right)$ and $\beta$ is real.

In $\$ 2$ we introduce the inverse operators $a^{-1}$ and $a^{\dagger-1}$ and discuss some of the commutation relations obeyed by them. In $\$ 3$ the desired eigenvalue equation is derived and we show that the state (1.12) is an eigenstate of the operator $\left(a^{\dagger-1} a\right)$. Also, we show that the squeezed first number state $\exp \left(\frac{1}{2} \xi a^{t^{2}}\right)|n=1\rangle$ is the eigenstate of another operator $\left(a a^{\dagger^{-1}}\right)$.

\section{Inverse operators}

We introduce inverse of the operators $\hat{a}$ and $\hat{a}^{\dagger}$ by their actions on the number states $|n\rangle$. These operators $a^{-1}$ and $a^{\dagger-1}$ are defined as [8]

$$
a^{-1}|n\rangle=\frac{1}{(n+1)^{1 / 2}}|n+1\rangle, \quad n=0,1,2, \ldots
$$


Squeezed vacuum as an eigenstate

$$
\begin{aligned}
a^{+-1}|n\rangle & =\frac{1}{\sqrt{n}}|n-1\rangle, & & n=1,2, \ldots, \\
& =0, & n & =0 .
\end{aligned}
$$

From (2.1) one can see that $a^{-1}$ behaves as a creation operator, while $a^{\dagger^{-1}}$ behaves as an annihilation operator. The matrix elements of these operators are given by the following relations:

$$
\begin{aligned}
& \left\langle m\left|a^{-1}\right| n\right\rangle=\frac{1}{(n+1)^{1 / 2}} \delta_{m, n+1}, \\
& \left\langle m\left|a^{+-1}\right| n\right\rangle=\frac{1}{\sqrt{n}}\left(1-\delta_{n, 0}\right) \delta_{m, n-1} .
\end{aligned}
$$

It is readily seen that $a^{-1}$ is the right inverse of $a$ and $a^{\dagger-1}$ is the left inverse of $a^{\dagger}$, i.e.

whereas

$$
a a^{-1}=a^{\dagger-1} a^{\dagger}=I
$$

$$
a^{-1} a=a^{\dagger} a^{\dagger-1}=I-|0\rangle\langle 0| .
$$

Here $|0\rangle\langle 0|$ is the projection operator on to the vacuum. We find that these operators satisfy the following commutation relations:

$$
\begin{aligned}
& {\left[a, a^{-1}\right]=\left[a^{\dagger-1}, a^{\dagger}\right]=|0\rangle\langle 0|,} \\
& {\left[a, a^{\dagger k}\right]=k a^{\dagger(k-1)},} \\
& {\left[a^{\dagger}, a^{k}\right]=-k a^{(k-1)},}
\end{aligned}
$$

where $k$ is any positive or negative integer.

\section{The eigenvalue equation}

As mentioned earlier, the squeezed vacuum [eq. (1.11)] may be expressed in the form:

$$
|\xi, 0\rangle=\left(1-|\xi|^{2}\right)^{1 / 4} \exp \left(\frac{1}{2} \xi a^{\dagger^{2}}\right)|0\rangle \text {. }
$$

We are, therefore, looking for an operator of which the state (3.1) is an eigenstate. Since the generation of a squeezed vacuum is essentially a two photon process, we consider the two photon annihilation operator $\left(a^{t^{-1}} a\right)$. From eq. (2.3) and the relation

$$
\left[a, \exp \left(\frac{1}{2} \xi a^{\dagger^{2}}\right)\right]=\xi a^{\dagger} \exp \left(\frac{1}{2} \xi a^{\dagger^{2}}\right),
$$

we find that

$$
\left[a^{\dagger^{-1}} a, \exp \left(\frac{1}{2} \xi a^{\dagger^{2}}\right)\right]=\xi \exp \left(\frac{1}{2} \xi a^{\dagger^{2}}\right)+\left[a^{\dagger^{-1}}, \exp \left(\frac{1}{2} \xi a^{\dagger^{2}}\right)\right] a .
$$

If we let (3.3) act on the vacuum, we obtain

$$
\left[a^{\dagger-1} a, \exp \left(\frac{1}{2} \xi a^{\dagger^{2}}\right)\right]|0\rangle=\xi \exp \left(\frac{1}{2} \xi a^{\dagger^{2}}\right)|0\rangle .
$$

Equation (3.4) is of interest since it can be expressed in the form

$$
a^{\dagger^{-1}} a\left[\exp \left(\frac{1}{2} \xi a^{\dagger^{2}}\right)|0\rangle\right]=\xi\left[\exp \left(\frac{1}{2} \xi a^{\dagger^{2}}\right)|0\rangle\right],
$$




\section{L Mehta et al}

which is an eigenvalue equation. Hence, we conclude that the squeezed vaccum (3.1), i.e., $\exp \left[\frac{1}{2} \xi a^{\dagger^{2}}\right]|0\rangle$ is an eigenstate of the operator $\left(\hat{a}^{\dagger^{-1}} a\right)$ with an eigenvalue $\xi$.

If we consider another two photon annihilation operator $\left(\hat{a} \hat{a}^{\dagger^{-1}}\right)$, we find that

$$
\left[\hat{a} \hat{a}^{\dagger^{-1}}, \exp \left(\frac{1}{2} \xi \hat{a}^{\dagger^{2}}\right)\right]=\xi \exp \left(\frac{1}{2} \xi \hat{a}^{\dagger^{2}}\right) \hat{a}^{\dagger} \hat{a}^{\dagger^{-1}}+a\left[\hat{a}^{\dagger-1}, \exp \left(\frac{1}{2} \xi \hat{a}^{\dagger^{2}}\right)\right] .
$$

On letting (3.6) operate on the first number state $|n=1\rangle$ we find that

$$
\left[\hat{a} \hat{a}^{\dagger^{-1}}, \exp \left(\frac{1}{2} \xi \hat{a}^{\dagger^{2}}\right)\right]|n=1\rangle=\xi \exp \left(\frac{1}{2} \xi \hat{a}^{\dagger^{2}}\right)|n=1\rangle \text {. }
$$

One can write immediately from this equation that

$$
a \hat{a}^{\dagger-1}\left[\exp \left(\frac{1}{2} \xi \hat{a}^{\dagger^{2}}\right)|n=1\rangle\right]=\xi\left[\exp \left(\frac{1}{2} \xi \hat{a}^{\dagger^{2}}\right)|n=1\rangle\right] .
$$

Hence, we conclude that the squeezed first number state, i.e., $\exp \left[\frac{1}{2} \xi a^{\dagger 2}\right]|n=1\rangle$, is an eigenstate of the operator $\left(\partial a^{\dagger-1}\right)$ with an eigenvalue $\xi$.

\section{References}

[1] R J Glauber, Phys. Rev., 131, 2766 (1963)

[2] D F Walls, Nature (London) 306, 141 (1983) and references therein

[3] R E Slusher, L W Hollberg, B Yurke, J C Mertz and J F Valley, Phys. Rev. Lett., 55, 2409 (1985)

[4] R M Shelby, M D Levenson, S H Perimutter, R G Devoe and D F Walls, Phys. Rev. Lett., 57, 691 (1986)

[5] L A Wu, H J Kimble, J L Hall and H Wu, Phys. Rev. Lett., 57, 2520 (1986)

[6] R M Shelby, M D Levenson, D F Walls, A Aspect and G J Milburn, Phys. Rev. A33, 4008 (1986)

[7] Anil K Roy and C L Mehta, J. Mod. Opt., 39, 1619 (1992)

[8] C L Mehta, Anil K Roy and G M Saxena, Phys. Rev. A46, 1565 (1992) 